Research Article

\title{
Nitrogen Gas Laser Filament Plasma Kinetic System Stability Analysis under Parameter Variation
}

\author{
Ofer Aluf Netanya* \\ "Corresponding Author: Ofer Aluf Netanya, Israel
}

Received: 22 July 2021; Accepted: 02 August 2021; Published: 25 August 2021

Citation: Ofer Aluf Netanya. Nitrogen Gas Laser Filament Plasma Kinetic System Stability Analysis under Parameter Variation. Journal of Analytical Techniques and Research 3 (2021): 46-59.

\begin{abstract}
A nitrogen laser is a gas laser operating in the ultraviolet range (typically $337.1 \mathrm{~nm}$ ) using molecular nitrogen as its gain medium, pumped by an electrical discharge. It is efficient sources for laserinduced fluorescence and photochemistry and general spectroscopy. There is a model for a singlepass, discharge type standoff nitrogen laser initiated by a femtosecond filament in nitrogen gas. A Nitrogen gas laser filament plasma kinetic system is described by the set of plasma kinetic equations which associated with rate equations for the population of the lasing levels and number of emitted photons. The change of concentration of different neural and ionic atomic and molecular species in the filament plasma is described by set of rate equation, $\mathrm{N}_{\mathrm{s}}$ is the density of species of type $s$, and $G_{s}, L_{s}$ are the relevant generation and loss rates. $\mathrm{T}, \mathrm{T}_{\mathrm{e}}$, and $\mathrm{T}_{\mathrm{vibr}}$ are gas, electronic, and vibrational temperatures, respectively. The concentration of system gas is not balanced and $\mathrm{T}_{\mathrm{e}} \approx \mathrm{T}_{\mathrm{vibr}}$. $\mathrm{T}$ parameters transform to $\mathrm{T} \rightarrow \mathrm{T}_{\mathrm{E}}+\Omega \cdot \sqrt{ } \mathrm{T} ; \mathrm{T}_{\mathrm{E}} \in \mathrm{N}, \Omega$

$\in \mathrm{R}$. We discuss the system stability and stability switching for different values of $\mathrm{T}_{\mathrm{E}}$ and $\Omega$ parameter.

There is a practical guideline that combines graphical information with analytical work to effectively study the local stability of models involving dependent parameters. The stability of a given steady state is determined by the graphs of some function of $\mathrm{T}_{\mathrm{E}}$ and $\Omega$ parameters.

Keywords: Nitrogen gas laser; Plasma; Filament; Electron temperatures; Vibrational temperature; Delay Differential Equation (DDE); Stability; Bifurcation; Orbit; Fixed point; Eigenvalue; Eigenstate

\section{Introduction}

In this article, we discuss the crucial subject of nitrogen gas laser filament plasma kinetic system stability and stability switching for different values 
of $T_{E}\left(T \rightarrow T_{E}+\Omega \cdot \sqrt{T}\right)$ and $\Omega$ parameter, where $T_{E} \in \mathbb{N} ; \Omega \in \mathbb{R}$. Lasing from molecular nitrogen is used in many scientific and industrial applications. The discharge pumped nitrogen laser, operating in a broad range of gas pressures, from several mill bars to the atmospheric pressure, and repetition rates from several hertz to several kilo hertz. It is robust source of high-power near-UV radiation. Achieving nitrogen lasing via remote excitation would pave the way to many potential applications. It is remotely initiated lasing from molecular gases by femtosecond filaments. A mid infrared femtosecond laser filament can introduce backward-directed lasing of molecular nitrogen via a resonant excitation transfer mechanism. The characterization of the filament ignited nitrogen laser, including the spatial and temporal properties of the generated UV emission and generation thresholds. A filament-assisted nitrogen laser is efficient as its conventional discharge-pumped counterpart. Mid-IR ultrashort laser pulses radically enhance fila mentation-assisted lasing of $\mathrm{N}_{2}$ relative to ultrashort pulses in the near-IR. We need a full analysis of multiple fila mentation. Another area is a remotely pumped $\mathrm{N}_{2}$ laser in the atmosphere and optimization of the pumping via electron- $\mathrm{N}_{2}$ collisions and evolving long-pulse light or microwave sources in combination with the femtosecond filament [1]. Filamentation is modeled by numerical solving the cylindrically symmetric nonlinear Schrodinger equation, which accounts for the impact of plasma dispersion and refraction, beam diffraction, Kerr, Raman, and plasma nonlinearities. We describe a change of concentration of different neutral and ionic atomic and molecular species in the filament plasma by the following set of rate equations:

$\frac{d\left[N_{s}\right]}{d t}=\left[G_{s}\right]-\left[L_{s}\right] ; \quad N_{s}$ is the density of species of type-s; $G_{s}$ and $L_{s}$ are the relevant generation and loss rates. Where the subscript $\mathrm{s}$ stands-for $e, A r^{+}, A r_{2}^{+}$

and

$N_{2}^{+}, N_{4}^{+}, N_{3}^{+}, N^{+}, N, N_{2}\left(A^{3} \Sigma_{u}\right), N_{2}\left(B^{3} \Pi_{g}\right), N_{2}\left(C^{3} \Pi_{u}\right) ;$ $\operatorname{Ar}^{*}\left(4^{3} P_{2}\right) ; N_{s}$ is the density of species of type$\mathrm{s}$; and $G_{s}, L_{s}$ are the relevant generation and loss rates. The rate equations are solved jointly with the equations for the electron temperature and the vibrational temperature of ground-state nitrogen molecules.

$\frac{3}{2} \cdot k \cdot N_{e} \cdot \frac{d T_{e}}{d t}=-\frac{3}{2} \cdot N_{e} \cdot k \cdot\left(T_{e}-T_{v i b r}\right) \cdot v_{*}$

$-\frac{3}{2} \cdot N_{e} \cdot k \cdot\left(T_{e}-T\right) \cdot\left[\delta_{N_{2}} \cdot\left(v_{N_{2}}+v_{N_{2}^{+}}+\frac{1}{2} \cdot v_{N_{4}^{+}}\right)\right.$

$\left.+\delta_{A_{r}} \cdot\left(v_{A_{r}}+v_{A_{r}^{+}}+\frac{1}{2} \cdot v_{A_{r_{2}}}\right)\right]+k_{e} \cdot N_{e} \cdot N_{A_{r_{r}}} \cdot I_{e x c}$

$-\left(T_{v i b r}-T\right) \times\left[\frac{1}{\tau_{V T, N_{2}}}+\left(\frac{N_{A_{r}}}{N_{2}}\right) / \tau_{V T, A_{r}}\right]$

$-\frac{3}{2} \cdot N_{e} \cdot k \cdot\left(T_{e}-T_{v i b r}\right) \cdot v_{*}-$ is the electron energy transfer to vibrational excitation of nitrogen-molecules. $T, T_{e}, T_{\text {vibr }}$ are gas, electronic, and vibrational temperatures, respectively. The concentration of system gas is not balanced and $T_{e} \approx T_{v i b r}$, and additionally there is a new behavior for $T$ parameter, $\rightarrow T_{\Xi}+\Omega \cdot \sqrt{T} ; T_{\Xi} \in \mathbb{N} ; \Omega \in \mathrm{R}$.

$-\frac{3}{2} \cdot N_{e} \cdot k \cdot\left(T_{e}-T\right) \cdot\left[\delta_{N_{2}} \cdot\left(v_{N_{2}}+v_{N_{2}^{+}}+\frac{1}{2} \cdot v_{N_{4}^{+}}\right)\right.$

$\left.+\delta_{A_{r}} \cdot\left(v_{A_{r}}+v_{A_{r}^{+}}+\frac{1}{2} \cdot v_{A_{r_{2}}}\right)\right]$ 
is the electron energy transfer in elastic and Coulombic collisions to translational energy of nitrogen and argon molecules, atoms, and ions. $k$ is the Boltzmann constant, $T, T_{e}, T_{v i b r}$ are gas, electronic, and vibrational temperatures, resp-ectively. $v_{s}$ are the frequencies of elastic collisions of electrons and corresponding species, $v_{*}$ is the frequency of inelastic collisions of electrons with $\mathrm{N}_{2}$ in the ground state which are responsible for vibrational heating; $\delta_{N_{2}}=\frac{2 \cdot m}{M_{N_{2}}} \quad \delta_{A_{r}}=\frac{2 \cdot m}{M_{A_{r}}}$; $k_{e}$ is the de excitation rate of excited argon atoms by electrons. Assumption: $G_{e}$ and $L_{e}$ are the relevant generation and loss rates, and they are very close in their values $G_{e}-L_{e} \rightarrow \varepsilon$ then $\frac{d N_{e}}{d t} \rightarrow \varepsilon$

$\frac{3}{2} \cdot k \cdot \frac{d}{d t}\left(N_{e} \cdot T_{e}\right)=\frac{3}{2} \cdot k \cdot \frac{d N_{e}}{d t} \cdot T_{e}$ $+\frac{3}{2} \cdot k \cdot N_{e} \cdot{\frac{d T_{e}}{d t}}^{\frac{d N_{e}}{d t} \rightarrow \varepsilon}=\frac{3}{2} \cdot k \cdot N_{e} \cdot \frac{d T_{e}}{d t}$

The reduced first-rate equation:

$$
\begin{aligned}
& \frac{3}{2} \cdot k \cdot N_{e} \cdot \frac{d T_{e}}{d t}=-\frac{3}{2} \cdot N_{e} \cdot k \cdot\left(T_{e}-T_{v i b r}\right) \cdot v_{*} \\
& -\frac{3}{2} \cdot N_{e} \cdot k \cdot\left(T_{e}-T\right) \cdot\left[\delta_{N_{2}} \cdot\left(v_{N_{2}}+v_{N_{2}^{+}}+\frac{1}{2} \cdot v_{N_{4}^{+}}\right)\right. \\
& \left.+\delta_{A_{r}} \cdot\left(v_{A_{r}}+v_{A_{r}^{+}}+\frac{1}{2} \cdot v_{A_{r_{2}^{+}}}\right)\right]+k_{e} \cdot N_{e} \cdot N_{A_{r^{*}}} \cdot I_{e x c}
\end{aligned}
$$

The second-rate equation:

$$
\begin{aligned}
& \frac{d T_{v i b r}}{d t}=\left(\frac{N_{e}}{N_{N_{2}}}\right) \cdot\left(T_{e}-T_{v i b r}\right) \cdot v_{*} \\
& -\left(T_{v i b r}-T\right) \times\left[\frac{1}{\tau_{V T, N_{2}}}+\left(\frac{N_{A_{r}}}{N_{2}}\right) / \tau_{V T, A_{r}}\right]
\end{aligned}
$$

Under the transformation $T \rightarrow T_{\Xi}+\Omega \cdot \sqrt{T} ; T_{\Xi} \in$
$\mathbb{N} ; \Omega \in \mathrm{R}$

We get the reduced rate equations:

$$
\begin{aligned}
& \frac{3}{2} \cdot k \cdot N_{e} \cdot \frac{d T_{e}}{d t}=-\frac{3}{2} \cdot N_{e} \cdot k \cdot\left(T_{e}-T_{v i b r}\right) \cdot v_{*} \\
& -\frac{3}{2} \cdot N_{e} \cdot k \cdot\left(T_{e}-T_{\Xi}-\Omega \cdot \sqrt{T}\right) \cdot\left[\delta _ { N _ { 2 } } \cdot \left(v_{N_{2}}\right.\right. \\
& \left.\left.+v_{N_{2}^{+}}+\frac{1}{2} \cdot v_{N_{4}^{+}}\right)+\delta_{A_{r}} \cdot\left(v_{A_{r}}+v_{A_{r}^{+}}+\frac{1}{2} \cdot v_{A_{r_{2}^{+}}}\right)\right] \\
& +k_{e} \cdot N_{e} \cdot N_{A_{r^{*}}} \cdot I_{e x c} \\
& \frac{d T_{v i b r}}{d t}=\left(\frac{N_{e}}{N_{N_{2}}}\right) \cdot\left(T_{e}-T_{v i b r}\right) \cdot v_{*} \\
& -\left(T_{v i b r}-T_{\Xi}-\Omega \cdot \sqrt{T}\right) \times\left[\frac{1}{\tau_{V T, N_{2}}}+\left(\frac{N_{A_{r}}}{N_{2}}\right) / \tau_{V T, A_{r}}\right]
\end{aligned}
$$

$\tau_{V T, N_{2}}$ and $\tau_{V T, A_{r}}$ are the vibrational relaxation times due to $\mathrm{N}_{2}-\mathrm{N}_{2}$ and $\mathrm{N}_{2}-\mathrm{A}_{\mathrm{r}}$ collisions, respectively. They are calculated by using the Landau-Teller approximation; $I_{e x c} \approx 11.8 \mathrm{eV}$ stands for the argon excitation energy into $A_{r^{*}}\left(4^{3} P_{2}\right)$ [2]. Rigorous bounds on the rate of energy exchange between vibrational and translational degrees of freedom are described by simple classical models of diatomic molecules. The outcomes are known as the elementary approximation introduced by Landau and Teller. The initial energy of electrons in the wake of the mid-IR laser pulse is taken equal to $1 \mathrm{eV}\left(\mathrm{T}_{\mathrm{e}}=0.66 \mathrm{eV}\right)$. The initial translational temperature of atoms and molecules in the gas mixture and the vibrational temperature of nitrogen molecules are set equal to $T=T_{V}=290 \mathrm{~K}$. We have two variables in our systems: $T_{e}$, electronic temperature and $T_{v i b r}$, vibrational temperature which change in time. We multiply the reduced first-rate equation's two sides by $\frac{2}{3 \cdot k \cdot N_{e}}$. 
Finally, we get the two reduced rate equations which include electron temperature and the vibrational temperature of ground-state nitrogenmolecules. $N_{2}$ is the nitrogen pressure ( 1 bar) and $N_{A_{r}}$ is Argon pressure. $v_{N_{2}}$ is frequency of $N_{2}, \quad v_{N_{2}^{+}}$is frequency of $N_{2}$ ions, $\quad v_{N_{4}^{+}}$is frequency of $N_{4}$ ions, $v_{A_{r}}$ is frequency of $A_{r}, v_{A_{r}^{+}}$ is frequency of $A_{r}$ ions, and $v_{A_{r_{2}^{+}}}$is frequency of $A_{r_{2}}$ ions.

$\frac{d T_{e}}{d t}=-\left(T_{e}-T_{v i b r}\right) \cdot v_{*}$

$-\left(T_{e}-T_{\Xi}-\Omega \cdot \sqrt{T}\right) \cdot\left[\delta_{N_{2}} \cdot\left(v_{N_{2}}\right.\right.$

$\left.\left.+v_{N_{2}^{+}}+\frac{1}{2} \cdot v_{N_{4}^{+}}\right)+\delta_{A_{r}} \cdot\left(v_{A_{r}}+v_{A_{r}^{+}}+\frac{1}{2} \cdot v_{A_{r_{2}^{+}}}\right)\right]$

$+k_{e} \cdot N_{A_{r^{*}}} \cdot I_{e x c} \cdot \frac{2}{3 \cdot k}$

$\frac{d T_{v i b r}}{d t}=\left(\frac{N_{e}}{N_{N_{2}}}\right) \cdot\left(T_{e}-T_{v i b r}\right) \cdot v_{*}$

$-\left(T_{v i b r}-T_{\Xi}-\Omega \cdot \sqrt{T}\right) \times\left[\frac{1}{\tau_{V T, N_{2}}}+\left(\frac{N_{A_{r}}}{N_{2}}\right) / \tau_{V T, A_{r}}\right]$

At fixed-points (equilibrium points):

$\frac{d T_{e}}{d t}=0 ; \frac{d T_{v i b r}}{d t}=0$

$-\left(T_{e}^{*}-T_{v i b r}^{*}\right) \cdot v_{*}$

$-\left(T_{e}^{*}-T_{\Xi}-\Omega \cdot \sqrt{T}\right) \cdot\left[\delta_{N_{2}} \cdot\left(v_{N_{2}}\right.\right.$

$\left.\left.+v_{N_{2}^{+}}+\frac{1}{2} \cdot v_{N_{4}^{+}}\right)+\delta_{A_{r}} \cdot\left(v_{A_{r}}+v_{A_{r}^{+}}+\frac{1}{2} \cdot v_{A_{r_{2}^{+}}}\right)\right]$

$+k_{e} \cdot N_{A_{r^{*}}} \cdot I_{e x c} \cdot \frac{2}{3 \cdot k}=0$

$\left(\frac{N_{e}}{N_{N_{2}}}\right) \cdot\left(T_{e}^{*}-T_{v i b r}^{*}\right) \cdot v_{*}-\left(T_{v i b r}^{*}-T_{\Xi}\right.$

$-\Omega \cdot \sqrt{T}) \times\left[\frac{1}{\tau_{V T, N_{2}}}+\left(\frac{N_{A_{r}}}{N_{2}}\right) / \tau_{V T, A_{r}}\right]=0$
We define the parameters functions:

$$
\begin{aligned}
& \psi_{1}=\left[\delta_{N_{2}} \cdot\left(v_{N_{2}}+v_{N_{2}^{+}}+\frac{1}{2} \cdot v_{N_{4}^{+}}\right)\right. \\
& \left.+\delta_{A_{r}} \cdot\left(v_{A_{r}}+v_{A_{r}^{+}}+\frac{1}{2} \cdot v_{A_{r_{2}^{+}}}\right)\right]
\end{aligned}
$$

$\psi_{2}=k_{e} \cdot N_{A_{r^{*}}} \cdot I_{e x c} \cdot \frac{2}{3 \cdot k}$

$\psi_{3}=\left[\frac{1}{\tau_{V T, N_{2}}}+\left(\frac{N_{A_{r}}}{N_{2}}\right) / \tau_{V T, A_{r}}\right]$

$-T_{e}^{*} \cdot v_{*}+T_{v i b r}^{*} \cdot v_{*}$

$-\left(T_{e}^{*}-T_{\Xi}-\Omega \cdot \sqrt{T}\right) \cdot \psi_{1}+\psi_{2}=0$

$\left(\frac{N_{e}}{N_{N_{2}}}\right) \cdot\left(T_{e}^{*}-T_{v i b r}^{*}\right) \cdot v_{*}$

$-\left(T_{v i b r}^{*}-T_{\Xi}-\Omega \cdot \sqrt{T}\right) \times \psi_{3}=0$

$T_{e}^{*}=T_{v i b r}^{*} \cdot\left(\frac{v_{*}}{\psi_{1}+v_{*}}\right)+\frac{T_{\Xi} \cdot \psi_{1}+\Omega \cdot \sqrt{T} \cdot \psi_{1}+\psi_{2}}{\psi_{1}+v_{*}}$

$T_{v i b r}^{*}=T_{e}^{*} \cdot\left(\frac{\left(\frac{N_{e}}{N_{N_{2}}}\right) \cdot v_{*}}{\left(\frac{N_{e}}{N_{N_{2}}}\right) \cdot v_{*}+\psi_{3}}\right)+\left(\frac{T_{\Xi} \cdot \psi_{3}+\Omega \cdot \sqrt{T} \cdot \psi_{3}}{\left(\frac{N_{e}}{N_{N_{2}}}\right) \cdot v_{*}+\psi_{3}}\right)$

Our system fixed points are:

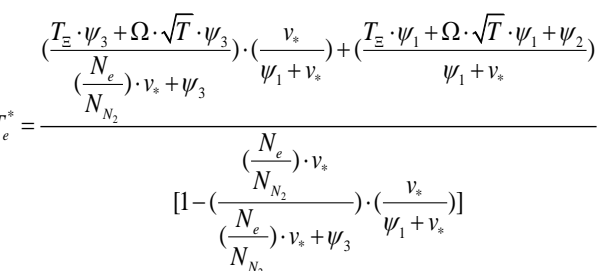

$$
\begin{aligned}
& T_{v i b r}^{*}=T_{e}^{*} \cdot\left(\frac{\left(\frac{N_{e}}{N_{N_{2}}}\right) \cdot v_{*}}{\left(\frac{N_{e}}{N_{N_{2}}}\right) \cdot v_{*}+\psi_{3}}\right)+\left(\frac{T_{\Xi} \cdot \psi_{3}+\Omega \cdot \sqrt{T} \cdot \psi_{3}}{\left(\frac{N_{e}}{N_{N_{2}}}\right) \cdot v_{*}+\psi_{3}}\right)
\end{aligned}
$$


We get the 3D function, $T_{e}^{*}$ (fixed electronic temperature value) and $T_{v i b r}^{*}$ (fixed vibrational temperature value) graphs as a function of $T_{\Xi}$ and $\Omega$ parameters. The initial translational temperature of atoms and molecules in the gas mixture and the vibrational temperature of nitrogen molecules are equal $T=T_{V}$ and $T=290{ }^{\circ} \mathrm{K} ; T_{V}=290{ }^{\circ} \mathrm{K}$ . We can set $T_{v}=T_{v i r b} ; n_{e}=N_{e}=n$ and $v_{e v}=v_{*}$ [3]. $T_{v}$ is vibrational temperature of $\mathrm{N}_{2}$ molecules, $T$ is the translational gas temperature, $\delta_{N_{2}}=\frac{2 \cdot m}{M_{N_{2}}}$, and $\delta_{A_{r}}=\frac{2 \cdot m}{M_{A_{r}}}$. The times of vibrational relaxation through $\mathrm{N} 2-\mathrm{N} 2\left(\tau_{V T, N_{2}}\right)$ and N2-A $\mathrm{A}_{\mathrm{r}}\left(\tau_{V T, A_{r}}\right)$ collisions are estimated using the Landau-Teller approximation:

$$
\begin{gathered}
\tau_{V T, N_{2}}=3.98 \times 10^{9} \cdot \frac{e^{\left(\frac{221.46}{T^{1 / 3}}\right)}}{N_{N_{2}}} \\
\tau_{V T, A_{r}}=1.73 \times 10^{9} \cdot \frac{e^{\left(\frac{239.5}{T^{1 / 3}}\right)}}{N_{A_{r}}}
\end{gathered}
$$

We define two-functions for the simplicity

$$
\xi_{1}\left(T, N_{N_{2}}\right)=\tau_{V T, N_{2}} \text { And } \xi_{2}\left(T, N_{A_{r}}\right)=\tau_{V T, A_{r}} \text {. A }
$$

vibrational energy transfer between diatomic molecules is important and the classical model in vibrational processes is Landau-Teller model. The model takes the population of each vibrational level to be given by the Boltzmann equilibrium distribution. The translation of gas temperature is almost constant for specified conditions and time intervals. A Resonance enhanced multi photon Ionization (REMPI) is an attractive for population inversion of molecular nitrogen in an $\mathrm{Ar}$ : $\mathrm{N}_{2}$ gas mixture. There is a big interest in a population inversion of molecular nitrogen in a REMPI pumped Ar: $\mathrm{N}_{2}$ gas mixture [3].

2. Nitrogen gas laser filament plasma kinetic system linearization and characteristic equation We suppose that $\left(T_{e}^{*}, T_{v i b r}^{*}\right)$ is a fixed point, $f_{1}\left(T_{e}^{*}, T_{v i b r}^{*}\right)=0 \quad$ and $f_{2}\left(T_{e}^{*}, T_{v i b r}^{*}\right)=0, \quad$ let $u_{1}=T_{e}-T_{e}^{*} ; u_{2}=T_{v i b r}-T_{v i b r}^{*} \quad$ denote the components of a small disturbance from the fixed point. To see whether the disturbance grows or decays, we need to derive differential equations for $u_{1}$ and $u_{2}$. Let's do the $u_{1}$-equation first:

$\frac{d u_{1}}{d t}=\frac{d T_{e}}{d t}$ since $T_{e}^{*}$ is constant and

$\frac{d u_{1}}{d t}=\frac{d T_{e}}{d t}=f_{1}\left(T_{e}^{*}+u_{1}, T_{v i b r}^{*}+u_{2}\right)$ by

substitution, then by using Taylor series expansion

$$
\begin{aligned}
& \frac{d u_{1}}{d t}=\frac{d T_{e}}{d t}=f_{1}\left(T_{e}^{*}, T_{v i b r}^{*}\right)+u_{1} \cdot \frac{\partial f_{1}}{\partial T_{e}} \\
& +u_{2} \cdot \frac{\partial f_{1}}{\partial T_{v i b r}}+O\left(u_{1}^{2}, u_{2}^{2}, u_{1} \cdot u_{2}\right)
\end{aligned}
$$

And since $f_{1}\left(T_{e}^{*}, T_{v i b r}^{*}\right)=0$ then

$$
\frac{d u_{1}}{d t}=\frac{d T_{e}}{d t}=u_{1} \cdot \frac{\partial f_{1}}{\partial T_{e}}+u_{2} \cdot \frac{\partial f_{1}}{\partial T_{v i b r}}+O\left(u_{1}^{2}, u_{2}^{2}, u_{1} \cdot u_{2}\right)
$$

To simplify the notation, we have written $\frac{\partial f_{1}}{\partial T_{e}}$ and $\frac{\partial f_{1}}{\partial T_{v i b r}}$, these partial derivatives are to be evaluated at the fixed point $\left(T_{e}^{*}, T_{v i b r}^{*}\right)$; thus they are numbers, not functions. The shorthand notation, $O\left(u_{1}^{2}, u_{2}^{2}, u_{1} \cdot u_{2}\right)$ denotes quadratic terms in $u_{1}$ 
and $u_{2}$ are small, these quadratic terms are extremely small $[7,8]$.

$$
\frac{d u_{2}}{d t}=\frac{d T_{v i b r}}{d t}=u_{1} \cdot \frac{\partial f_{2}}{\partial T_{e}}+u_{2} \cdot \frac{\partial f_{2}}{\partial T_{v i b r}}+O\left(u_{1}^{2}, u_{2}^{2}, u_{1} \cdot u_{2}\right)
$$

Hence the disturbance $\left(u_{1}, u_{2}\right)$ evolves according to

$$
\left(\begin{array}{c}
\frac{d u_{1}}{d t} \\
\frac{d u_{2}}{d t}
\end{array}\right)=\left(\begin{array}{cc}
\frac{\partial f_{1}}{\partial T_{e}} & \frac{\partial f_{1}}{\partial T_{v i b r}} \\
\frac{\partial f_{2}}{\partial T_{e}} & \frac{\partial f_{2}}{\partial T_{v i b r}}
\end{array}\right) \cdot\left(\begin{array}{l}
u_{1} \\
u_{2}
\end{array}\right)+\text { quadratic terms } .
$$

The matrix $A=\left(\begin{array}{ll}\frac{\partial f_{1}}{\partial T_{e}} & \frac{\partial f_{1}}{\partial T_{v i b r}} \\ \frac{\partial f_{2}}{\partial T_{e}} & \frac{\partial f_{2}}{\partial T_{v i b r}}\end{array}\right)_{\left(T_{e}^{*}, T_{v i b r}^{*}\right)} \quad$ is called the Jacobian matrix at the fixed point $\left(T_{e}^{*}, T_{v i b r}^{*}\right)$. It is multivariable analog to $f_{1}^{\prime}\left(T_{e}^{*}\right)$ [7][8]. Since the quadratic terms are tiny, we neglect them altogether. We obtain the linearized system:

$$
\begin{aligned}
& \left(\begin{array}{c}
\frac{d u_{1}}{d t} \\
\frac{d u_{2}}{d t}
\end{array}\right)=\left(\begin{array}{cc}
\frac{\partial f_{1}}{\partial T_{e}} & \frac{\partial f_{1}}{\partial T_{v i b r}} \\
\frac{\partial f_{2}}{\partial T_{e}} & \frac{\partial f_{2}}{\partial T_{v i b r}}
\end{array}\right) \cdot\left(\begin{array}{l}
u_{1} \\
u_{2}
\end{array}\right) \\
& f_{1}=f_{1}\left(T_{e}, T_{v i b r}\right)=-\left(T_{e}-T_{v i b r}\right) \cdot v_{*} \\
& -\left(T_{e}-T_{\Xi}-\Omega \cdot \sqrt{T}\right) \cdot\left[\delta _ { N _ { 2 } } \cdot \left(v_{N_{2}}\right.\right. \\
& \left.\left.+v_{N_{2}^{+}}+\frac{1}{2} \cdot v_{N_{4}^{+}}\right)+\delta_{A_{r}} \cdot\left(v_{A_{r}}+v_{A_{r}^{+}}+\frac{1}{2} \cdot v_{A_{r_{2}^{+}}}\right)\right] \\
& +k_{e} \cdot N_{A_{r}^{*}} \cdot I_{e x c} \cdot \frac{2}{3 \cdot k}
\end{aligned}
$$

$$
\begin{aligned}
& f_{2}=f_{2}\left(T_{e}, T_{v i b r}\right)=\left(\frac{N_{e}}{N_{N_{2}}}\right) \cdot\left(T_{e}-T_{v i b r}\right) \cdot v_{*} \\
& -\left(T_{v i b r}-T_{\Xi}-\Omega \cdot \sqrt{T}\right) \times\left[\frac{1}{\tau_{V T, N_{2}}}+\left(\frac{N_{A_{r}}}{N_{2}}\right) / \tau_{V T, A_{r}}\right]
\end{aligned}
$$

Function $\quad f_{2}\left(T_{e}, T_{v i b r}\right)$ includes functions $\xi_{1}\left(T, N_{N_{2}}\right)$ and $\xi_{2}\left(T, N_{A_{r}}\right)$.

$$
\begin{aligned}
& f_{2}=f_{2}\left(T_{e}, T_{v i b r}\right)=\left(\frac{N_{e}}{N_{N_{2}}}\right) \cdot\left(T_{e}-T_{v i b r}\right) \cdot v_{*} \\
& -\left(T_{v i b r}-T_{\Xi}-\Omega \cdot \sqrt{T}\right) \times\left[\frac{1}{\xi_{1}\left(T, N_{N_{2}}\right)}\right. \\
& \left.+\left(\frac{N_{A_{r}}}{N_{2}}\right) / \xi_{2}\left(T, N_{A_{r}}\right)\right]
\end{aligned}
$$

$$
\begin{aligned}
& \frac{\partial f_{1}}{\partial T_{e}}=-v_{*}-\left[\delta_{N_{2}} \cdot\left(v_{N_{2}}+v_{N_{2}^{+}}+\frac{1}{2} \cdot v_{N_{4}^{+}}\right)\right. \\
& \left.+\delta_{A_{r}} \cdot\left(v_{A_{r}}+v_{A_{r}^{+}}+\frac{1}{2} \cdot v_{A_{r_{2}^{+}}}\right)\right] ; \frac{\partial f_{1}}{\partial T_{v i b r}}=v_{*} \\
& \frac{\partial f_{2}}{\partial T_{e}}=\left(\frac{N_{e}}{N_{N_{2}}}\right) \cdot v_{*}
\end{aligned}
$$$$
\left.+\left(\frac{N_{A_{r}}}{N_{2}}\right) / \xi_{2}\left(T, N_{A_{r}}\right)\right]
$$

$$
A=\left(\begin{array}{cc}
-v_{*}-\left[\delta _ { N _ { 2 } } \cdot \left(v_{N_{2}}\right.\right. & \\
\left.+v_{N_{2}^{+}}+\frac{1}{2} \cdot v_{N_{4}^{+}}\right) & v_{*} \\
+\delta_{A_{r}} \cdot\left(v_{A_{r}}+v_{A_{r}^{+}}\right. & \\
\left.\left.+\frac{1}{2} \cdot v_{A_{r^{+}}}\right)\right] & -\left(\frac{N_{e}}{N_{N_{2}}}\right) \cdot v_{*} \\
\left(\frac{N_{e}}{N_{N_{2}}}\right) \cdot v_{*} & -\left[\frac{1}{\xi_{1}\left(T, N_{N_{2}}\right)}\right. \\
& \left.+\left(\frac{N_{A_{r}}}{N_{2}}\right) / \xi_{2}\left(T, N_{A_{r}}\right)\right]
\end{array}\right)_{\left(T_{e}^{*}, T_{v i r r}^{*}\right)}
$$


The eigenvalues of the matrix A are given by the characteristic equation $\operatorname{det}(A-\lambda \cdot I)=0$, where $I$ is the identity matrix. For our $2 \times 2$ matrix the characteristic equation becomes:

$$
\operatorname{det}\left(\begin{array}{cc}
-v_{*}-\left[\delta _ { N _ { 2 } } \cdot \left(v_{N_{2}}\right.\right. & \\
\left.+v_{N_{2}^{+}}+\frac{1}{2} \cdot v_{N_{4}^{+}}\right) & v_{*} \\
+\delta_{A_{r}} \cdot\left(v_{A_{r}}+v_{A_{r}^{+}}\right. & -\left(\frac{N_{e}}{N_{N_{2}}}\right) \cdot v_{*} \\
\left.\left.+\frac{1}{2} \cdot v_{A_{r^{+}}}\right)\right]-\lambda & -\left[\frac{1}{\xi_{1}\left(T, N_{N_{2}}\right)}\right. \\
\left(\frac{N_{e}}{N_{N_{2}}}\right) \cdot v_{*} & \left.+\left(\frac{N_{A_{r}}}{N_{2}}\right) / \xi_{2}\left(T, N_{A_{r}}\right)\right]-\lambda
\end{array}\right)=0
$$

For simplicity we define two global parameters: $\Upsilon_{1}, \Upsilon_{2}$

$$
\begin{aligned}
& \Upsilon_{1}=-v_{*}-\left[\delta_{N_{2}} \cdot\left(v_{N_{2}}+v_{N_{2}^{+}}+\frac{1}{2} \cdot v_{N_{4}^{+}}\right)\right. \\
& \left.+\delta_{A_{r}} \cdot\left(v_{A_{r}}+v_{A_{r}^{+}}+\frac{1}{2} \cdot v_{A_{r_{2}^{+}}}\right)\right]
\end{aligned}
$$

$$
\begin{aligned}
& \Upsilon_{2}=-\left(\frac{N_{e}}{N_{N_{2}}}\right) \cdot v_{*}-\left[\frac{1}{\xi_{1}\left(T, N_{N_{2}}\right)}\right. \\
& \left.+\left(\frac{N_{A_{r}}}{N_{2}}\right) / \xi_{2}\left(T, N_{A_{r}}\right)\right]
\end{aligned}
$$

$\operatorname{det}\left(\begin{array}{cc}\Upsilon_{1}-\lambda & v_{*} \\ \left(\frac{N_{e}}{N_{N_{2}}}\right) \cdot v_{*} & \Upsilon_{2}-\lambda\end{array}\right)=0$

$$
\left(\Upsilon_{1}-\lambda\right) \cdot\left(\Upsilon_{2}-\lambda\right)-\left(\frac{N_{e}}{N_{N_{2}}}\right) \cdot v_{*}^{2}=0
$$

Expanding the determinant yields:

$$
\lambda^{2}-\lambda \cdot\left(\Upsilon_{1}+\Upsilon_{2}\right)+\left(\Upsilon_{1} \cdot \Upsilon_{2}-\frac{N_{e}}{N_{N_{2}}} \cdot v_{*}^{2}\right)=0
$$

Another way to get the system characteristic equation and explore stability is by adding to coordinates $\left[T_{e} T_{v i b r}\right]$ arbitrarily small increments of exponential form $\left[t_{e} t_{v i b r}\right] \cdot e^{\lambda \cdot t}$, and retaining the first order terms in $T_{e} T_{v i b r}$. The system of two homogeneous equations leads to a polynomial characteristics equation in the eigenvalues $\lambda$. Nitrogen gas laser filament plasma kinetic system $T_{e}$, electronic temperature and $T_{v i b r}$, vibrational temperature fixed values with arbitrarily small increments of exponential form $\left[t_{e} t_{v i b r}\right] \cdot e^{\lambda \cdot t}$ are $T_{e}=T_{e}^{*}+t_{e} \cdot e^{\lambda \cdot t} ; T_{v i b r}=T_{v i b r}^{*}+t_{v i b r} \cdot e^{\lambda \cdot t}$.

We choose these expressions for our $T_{e}(t), T_{v i b r}(t)$ as small $\left[t_{e} t_{v i b r}\right]$ from the system fixed points at time $t=0$;

$$
T_{e}(t=0)=T_{e}^{*}+t_{e} ; T_{v i b r}(t=0)=T_{v i b r}^{*}
$$

$$
\frac{d T_{e}}{d t}=t_{e} \cdot \lambda \cdot e^{\lambda \cdot t} ; \frac{d T_{v i b r}}{d t}=t_{v i b r} \cdot \lambda \cdot e^{\lambda \cdot t}
$$

$$
\begin{aligned}
& \frac{d T_{e}}{d t}=-\left(T_{e}-T_{v i b r}\right) \cdot v_{*} \\
& -\left(T_{e}-T_{\Xi}-\Omega \cdot \sqrt{T}\right) \cdot\left[\delta _ { N _ { 2 } } \cdot \left(v_{N_{2}}\right.\right. \\
& \left.\left.+v_{N_{2}^{+}}+\frac{1}{2} \cdot v_{N_{4}^{+}}\right)+\delta_{A_{r}} \cdot\left(v_{A_{r}}+v_{A_{r}^{+}}+\frac{1}{2} \cdot v_{A_{r_{2}^{+}}}\right)\right] \\
& +k_{e} \cdot N_{A_{r^{*}}} \cdot I_{e x c} \cdot \frac{2}{3 \cdot k}
\end{aligned}
$$




$$
\begin{aligned}
& t_{e} \cdot \lambda \cdot e^{\lambda \cdot t}=-\left(T_{e}^{*}+t_{e} \cdot e^{\lambda \cdot t}-T_{v i b r}^{*}-t_{v i b r} \cdot e^{\lambda \cdot t}\right) \cdot v_{*} \\
& -\left(T_{e}^{*}+t_{e} \cdot e^{\lambda \cdot t}-T_{\Xi}-\Omega \cdot \sqrt{T}\right) \cdot\left[\delta _ { N _ { 2 } } \cdot \left(v_{N_{2}}\right.\right. \\
& \left.\left.+v_{N_{2}^{+}}+\frac{1}{2} \cdot v_{N_{4}^{+}}\right)+\delta_{A_{r}} \cdot\left(v_{A_{r}}+v_{A_{r}^{+}}+\frac{1}{2} \cdot v_{A_{r_{2}^{+}}}\right)\right] \\
& +k_{e} \cdot N_{A_{r^{*}}} \cdot I_{e x c} \cdot \frac{2}{3 \cdot k}
\end{aligned}
$$$$
\begin{aligned}
& \left\{-v_{*}-\left[\delta_{N_{2}} \cdot\left(v_{N_{2}}+v_{N_{2}^{+}}+\frac{1}{2} \cdot v_{N_{4}^{+}}\right)\right.\right. \\
& \left.\left.+\delta_{A_{r}} \cdot\left(v_{A_{r}}+v_{A_{r}^{+}}+\frac{1}{2} \cdot v_{{r_{2}}_{2}^{+}}\right)\right]-\lambda\right\} \cdot t_{e}+t_{v i b r} \cdot v_{*}=0
\end{aligned}
$$

$\frac{d T_{v i b r}}{d t}=\left(\frac{N_{e}}{N_{N_{2}}}\right) \cdot\left(T_{e}-T_{v i b r}\right) \cdot v_{*}$

$-\left(T_{v i b r}-T_{\Xi}-\Omega \cdot \sqrt{T}\right) \times\left[\frac{1}{\tau_{V T, N_{2}}}+\left(\frac{N_{A_{r}}}{N_{2}}\right) / \tau_{V T, A_{r}}\right]$

$t_{e} \cdot \lambda \cdot e^{\lambda \cdot t}=-\left(T_{e}^{*}-T_{v i b r}^{*}\right) \cdot v_{*}-\left(t_{e}-t_{v i b r}\right) \cdot v_{*} \cdot e^{\lambda \cdot t}$

$-\left(T_{e}^{*}-T_{\Xi}-\Omega \cdot \sqrt{T}\right) \cdot\left[\delta_{N_{2}} \cdot\left(v_{N_{2}}+v_{N_{2}^{+}}+\frac{1}{2} \cdot v_{N_{4}^{+}}\right)\right.$

$\left.+\delta_{A_{r}} \cdot\left(v_{A_{r}}+v_{A_{r}^{+}}+\frac{1}{2} \cdot v_{A_{r_{2}^{+}}}\right)\right]-t_{e} \cdot\left[\delta_{N_{2}} \cdot\left(v_{N_{2}}\right.\right.$

$\left.\left.+v_{N_{2}^{+}}+\frac{1}{2} \cdot v_{N_{4}^{+}}\right)+\delta_{A_{r}} \cdot\left(v_{A_{r}}+v_{A_{r}^{+}}+\frac{1}{2} \cdot v_{A_{r_{2}^{+}}}\right)\right] \cdot e^{\lambda \cdot t}$

$t_{v i b r} \cdot \lambda \cdot e^{\lambda \cdot t}=\left(\frac{N_{e}}{N_{N_{2}}}\right) \cdot\left(T_{e}^{*}+t_{e} \cdot e^{\lambda \cdot t}\right.$

$\left.-T_{v i b r}^{*}-t_{v i b r} \cdot e^{\lambda \cdot t}\right) \cdot v_{*}-\left(T_{v i b r}^{*}+t_{v i b r} \cdot e^{\lambda \cdot t}\right.$

$+k_{e} \cdot N_{A_{r^{*}}} \cdot I_{e x c} \cdot \frac{2}{3 \cdot k}$

$\left.-T_{\Xi}-\Omega \cdot \sqrt{T}\right) \times\left[\frac{1}{\tau_{V T, N_{2}}}+\left(\frac{N_{A_{r}}}{N_{2}}\right) / \tau_{V T, A_{r}}\right]$

$$
t_{v i b r} \cdot \lambda \cdot e^{\lambda \cdot t}=\left(\frac{N_{e}}{N_{N_{2}}}\right) \cdot\left(T_{e}^{*}-T_{v i b r}^{*}\right) \cdot v_{*}
$$$$
t_{e} \cdot \lambda \cdot e^{\lambda \cdot t}=-\left(T_{e}^{*}-T_{v i b r}^{*}\right) \cdot v_{*}
$$$$
-\left(T_{e}^{*}-T_{\Xi}-\Omega \cdot \sqrt{T}\right) \cdot\left[\delta_{N_{2}} \cdot\left(v_{N_{2}}+v_{N_{2}^{+}}+\frac{1}{2} \cdot v_{N_{4}^{+}}\right)\right.
$$$$
\left.+\delta_{A_{r}} \cdot\left(v_{A_{r}}+v_{A_{r}^{+}}+\frac{1}{2} \cdot v_{A_{r_{2}^{+}}}\right)\right]+k_{e} \cdot N_{A_{r^{*}}} \cdot I_{e x c} \cdot \frac{2}{3 \cdot k}
$$$$
-\left(t_{e}-t_{v i b r}\right) \cdot v_{*} \cdot e^{\lambda \cdot t}-t_{e} \cdot\left[\delta_{N_{2}} \cdot\left(v_{N_{2}}+v_{N_{2}^{+}}+\frac{1}{2} \cdot v_{N_{4}^{+}}\right)\right.
$$$$
\left.+\delta_{A_{r}} \cdot\left(v_{A_{r}}+v_{A_{r}^{+}}+\frac{1}{2} \cdot v_{A_{r_{2}^{+}}}\right)\right] \cdot e^{\lambda \cdot t}
$$

At fixed points:

$$
\begin{aligned}
& -\left(T_{e}^{*}-T_{v i b r}^{*}\right) \cdot v_{*}-\left(T_{e}^{*}-T_{\Xi}-\Omega \cdot \sqrt{T}\right) \\
& \cdot\left[\delta_{N_{2}} \cdot\left(v_{N_{2}}+v_{N_{2}^{+}}+\frac{1}{2} \cdot v_{N_{4}^{+}}\right)\right. \\
& \left.+\delta_{A_{r}} \cdot\left(v_{A_{r}}+v_{A_{r}^{+}}+\frac{1}{2} \cdot v_{A_{r_{2}^{+}}}\right)\right] \\
& +k_{e} \cdot N_{A_{r^{*}}} \cdot I_{\text {exc }} \cdot \frac{2}{3 \cdot k}=0
\end{aligned}
$$

$$
t_{v i b r} \cdot \lambda \cdot e^{\lambda \cdot t}=\left(\frac{N_{e}}{N_{N_{2}}}\right) \cdot\left(T_{e}^{*}-T_{v i b r}^{*}\right) \cdot v_{*}
$$

$$
\begin{aligned}
& -\left(T_{v i b r}^{*}-T_{\Xi}-\Omega \cdot \sqrt{T}\right) \times\left[\frac{1}{\tau_{V T, N_{2}}}+\left(\frac{N_{A_{r}}}{N_{2}}\right) / \tau_{V T, A_{r}}\right] \\
& -t_{v i b r} \times\left[\frac{1}{\tau_{V T, N_{2}}}+\left(\frac{N_{A_{r}}}{N_{2}}\right) / \tau_{V T, A_{r}}\right] \cdot e^{\lambda \cdot t} \\
& +\left(\frac{N_{e}}{N_{N_{2}}}\right) \cdot\left(t_{e}-t_{v i b r}\right) \cdot v_{*} \cdot e^{\lambda \cdot t}
\end{aligned}
$$

At fixed points:

$$
\begin{aligned}
& t_{e} \cdot \lambda=-\left(t_{e}-t_{v i b r}\right) \cdot v_{*}-t_{e} \cdot\left[\delta _ { N _ { 2 } } \cdot \left(v_{N_{2}}\right.\right. \\
& \left.\left.+v_{N_{2}^{+}}+\frac{1}{2} \cdot v_{N_{4}^{+}}\right)+\delta_{A_{r}} \cdot\left(v_{A_{r}}+v_{A_{r}^{+}}+\frac{1}{2} \cdot v_{A_{r_{2}^{+}}}\right)\right]
\end{aligned}
$$

$$
\begin{aligned}
& \left(\frac{N_{e}}{N_{N_{2}}}\right) \cdot\left(T_{e}^{*}-T_{v i b r}^{*}\right) \cdot v_{*}-\left(T_{v i b r}^{*}-T_{\Xi}\right. \\
& -\Omega \cdot \sqrt{T}) \times\left[\frac{1}{\tau_{V T, N_{2}}}+\left(\frac{N_{A_{r}}}{N_{2}}\right) / \tau_{V T, A_{r}}\right]=0
\end{aligned}
$$




$$
\begin{aligned}
& t_{v i b r} \cdot \lambda=-t_{v i b r} \times\left[\frac{1}{\tau_{V T, N_{2}}}+\left(\frac{N_{A_{r}}}{N_{2}}\right) / \tau_{V T, A_{r}}\right] \\
& +\left(\frac{N_{e}}{N_{N_{2}}}\right) \cdot\left(t_{e}-t_{v i b r}\right) \cdot v_{*} \\
& \frac{N_{e}}{N_{N_{2}}} \cdot v_{*} \cdot t_{e}-\left\{\frac{N_{e}}{N_{N_{2}}} \cdot v_{*}+\left[\frac{1}{\tau_{V T, N_{2}}}\right.\right. \\
& \left.\left.+\left(\frac{N_{A_{r}}}{N_{2}}\right) / \tau_{V T, A_{r}}\right]\right\} \cdot t_{v i b r}-t_{v i b r} \cdot \lambda=0
\end{aligned}
$$

The small increment Jacobian of our Nitrogen gas laser filament plasma kinetic system is as follow:

$$
\left\{-v_{*}-\left[\delta_{N_{2}} \cdot\left(v_{N_{2}}+v_{N_{2}^{+}}+\frac{1}{2} \cdot v_{N_{4}^{+}}\right)\right.\right.
$$$$
\left.\left.+\delta_{A_{r}} \cdot\left(v_{A_{r}}+v_{A_{r}^{+}}+\frac{1}{2} \cdot v_{A_{r_{2}^{+}}}\right)\right]-\lambda\right\} \cdot t_{e}+t_{v i b r} \cdot v_{*}=0
$$

$$
\frac{N_{e}}{N_{N_{2}}} \cdot v_{*} \cdot t_{e}-\left\{\frac{N_{e}}{N_{N_{2}}} \cdot v_{*}+\left[\frac{1}{\tau_{V T, N_{2}}}\right.\right.
$$$$
\left.\left.+\left(\frac{N_{A_{r}}}{N_{2}}\right) / \tau_{V T, A_{r}}\right]\right\} \cdot t_{v i b r}-t_{v i b r} \cdot \lambda=0
$$

$$
\left(\begin{array}{cc}
-v_{*}-\left[\delta _ { N _ { 2 } } \cdot \left(v_{N_{2}}\right.\right. & \\
\left.+v_{N_{2}^{+}}+\frac{1}{2} \cdot v_{N_{4}^{+}}\right) & v_{*} \\
+\delta_{A_{r}} \cdot\left(v_{A_{r}}+v_{A_{r}^{+}}\right. & \\
\left.+\frac{1}{2} \cdot v_{A_{r_{t}}}\right]-\lambda & \\
\frac{N_{e}}{N_{N_{2}}} \cdot v_{*} & -\left\{\frac{N_{e}}{N_{N_{2}}} \cdot v_{*}\right. \\
& +\left[\frac{1}{\tau_{V T, N_{2}}}+\left(\frac{N_{A_{r}}}{N_{2}}\right)\right. \\
& \left.I \tau_{V T, A_{r}}\right]-\lambda
\end{array}\right) \cdot\left(\begin{array}{l}
t_{e} \\
t_{v i b r}
\end{array}\right)=\left(\begin{array}{l}
0 \\
0
\end{array}\right)
$$

$$
A=\left(\begin{array}{cc}
-v_{*}-\left[\delta _ { N _ { 2 } } \cdot \left(v_{N_{2}}\right.\right. & \\
\left.+v_{N_{2}^{+}}+\frac{1}{2} \cdot v_{N_{4}^{+}}\right) & \multicolumn{1}{c}{v_{*}} \\
+\delta_{A_{r}} \cdot\left(v_{A_{r}}+v_{A_{r}^{+}}\right. & \\
\left.\left.+\frac{1}{2} \cdot v_{A_{r^{+}}}\right)\right] & -\left\{\frac{N_{e}}{N_{N_{2}}} \cdot v_{*}\right. \\
\frac{N_{e}}{N_{N_{2}}} \cdot v_{*} & +\left[\frac{1}{\tau_{V T, N_{2}}}+\left(\frac{N_{A_{r}}}{N_{2}}\right)\right. \\
& \left.\left./ \tau_{V T, A_{r}}\right]\right\}
\end{array}\right)
$$

For simplicity we define two global parameters:

$$
l_{1}, l_{2}
$$$$
l_{1}=-v_{*}-\left[\delta_{N_{2}} \cdot\left(v_{N_{2}}+v_{N_{2}^{+}}+\frac{1}{2} \cdot v_{N_{4}^{+}}\right)\right.
$$$$
\left.+\delta_{A_{r}} \cdot\left(v_{A_{r}}+v_{A_{r}^{+}}+\frac{1}{2} \cdot v_{A_{r_{2}^{+}}}\right)\right]
$$

$$
\iota_{2}=-\left\{\frac{N_{e}}{N_{N_{2}}} \cdot v_{*}+\left[\frac{1}{\tau_{V T, N_{2}}}+\left(\frac{N_{A_{r}}}{N_{2}}\right)\right.\right.
$$

$\left.\left.\mid \tau_{V T, A_{r}}\right]\right\}$

$$
\begin{aligned}
& \operatorname{det}(A-\lambda \cdot I)=0 \\
& \operatorname{det}\left(\begin{array}{cc}
l_{1}-\lambda & v_{*} \\
\frac{N_{e}}{N_{N_{2}}} \cdot v_{*} & \iota_{2}-\lambda
\end{array}\right)=0
\end{aligned}
$$




$$
\left(l_{1}-\lambda\right) \cdot\left(l_{2}-\lambda\right)-\frac{N_{e}}{N_{N_{2}}} \cdot v_{*}^{2}=0
$$

The system characteristic equation is

$$
\lambda^{2}-\left(l_{1}+l_{2}\right) \cdot \lambda+\left(l_{1} \cdot l_{2}-\frac{N_{e}}{N_{N_{2}}} \cdot v_{*}^{2}\right)=0
$$

$t_{e}$ is system electronic temperature small increment and $t_{v i b r}$ is system vibrational temperature small increment. The electron temperature of plasma can be several orders of magnitude higher than the temperature of the neutral species or of the ions. Laser-induced plasma has very high electron temperature; the photo-thermoelectric effect is due to a local increase of the electron temperature induced by the laser illumination. Electron temp-eratures are measured by firing a laser beam into the plasma and detecting the light scattered by the electrons. Electron temperature in defined to be a temperature of free electrons. The electron temp-erature of the Laser Induce Plasma (LIP) is mea-sured by using Boltzmann equation

$\ln \left(\frac{I \cdot \lambda_{l w}}{g \cdot A}\right)=-\frac{E}{k \cdot T_{\text {elec }}}+C$, where, $I$ is the relative intensity of the line emission, $\lambda_{l w}$ is the line wavelength, $g$ is the statistical weight of the upper level, $A$ is the transition probability, $E$ is the energy pf the upper level, $k$ is the Boltzmann constant, $T_{\text {elec }}$ is the electron temperature of the Laser Induce Plasma (LIP), and $C$ is a constant. By introducing laser supported radiation wave model, the dependent of the electron temperature $T_{\text {elec }}$ is expressed.

$T_{\text {elec }} \approx\left(\frac{I_{\text {rad }} \cdot e_{i}}{\sigma \cdot \varepsilon_{\text {Plasma }} \cdot E_{\text {scal }}}\right)^{0.18}$, where, $I_{\text {rad }}$ is the laser irradiance, $\sigma$ is the Boltzmann constant, $\varepsilon_{\text {Plasma }}$ is the plasma emissivity, $e_{i}$ is the internal energy (energy per unit mass), of the plasma at the ignition point, and $E_{\text {scal }}$ is a scaling constant.

The vibrational and rotational temperatures in the supersonic flow of a gas-dynamic laser are determined simultaneously by using spectral gain measurement techniques (over a number of rota-tional lines). The vibrational and rotational temp-erature and, consequently, the optical energy in the flow are reported for two different gas-dynamic lasers having significantly different area ratio nozzles and varying stagnation temperatures $[9,10]$.

3. Nitrogen gas laser filament plasma kinetic system stability and stability switching

Our Nitrogen gas laser filament plasma kinetic system's characteristic equation is

$$
\lambda^{2}-\left(l_{1}+l_{2}\right) \cdot \lambda+\left(l_{1} \cdot l_{2}-\frac{N_{e}}{N_{N_{2}}} \cdot v_{*}^{2}\right)=0
$$

The eigenvalue of a matrix $A$ are given by the characteristic equation $\operatorname{det}(A-\lambda \cdot I)=0$, where $I$ is the identity matrix.

$A=\left(\begin{array}{cc}l_{1} & v_{*} \\ \frac{N_{e}}{N_{N_{2}}} \cdot v_{*} & \iota_{2}\end{array}\right) ; \operatorname{det}(A-\lambda \cdot I)=0$

$\operatorname{det}\left(\begin{array}{cc}l_{1}-\lambda & v_{*} \\ \frac{N_{e}}{N_{N_{2}}} \cdot v_{*} & l_{2}-\lambda\end{array}\right)=0$

We define $\wp_{1}=\operatorname{trace}(A) ; \wp_{2}=\operatorname{det}(A)$, the $\wp_{1}$ is equal 


$$
\begin{aligned}
& \wp_{1}=\operatorname{trace}(A)=l_{1}+l_{2}=-v_{*}-\left[\delta _ { N _ { 2 } } \cdot \left(v_{N_{2}}+v_{N_{2}^{+}}\right.\right. \\
& \left.\left.+\frac{1}{2} \cdot v_{N_{4}^{+}}\right)+\delta_{A_{r}} \cdot\left(v_{A_{r}}+v_{A_{r}^{+}}+\frac{1}{2} \cdot v_{A_{r_{2}}}\right)\right]-\left\{\frac{N_{e}}{N_{N_{2}}} \cdot v_{*}\right. \\
& \left.+\left[\frac{1}{\tau_{V T, N_{2}}}+\left(\frac{N_{A_{r}}}{N_{2}}\right) / \tau_{V T, A_{r}}\right]\right\} \\
& \xi_{1}\left(T, N_{N_{2}}\right)=\tau_{V T, N_{2}} ; \xi_{2}\left(T, N_{A_{r}}\right)=\tau_{V T, A_{r}} \\
& \wp_{1}=\operatorname{trace}(A)=l_{1}+l_{2}=-v_{*}-\left[\delta _ { N _ { 2 } } \cdot \left(v_{N_{2}}+v_{N_{2}^{+}}\right.\right. \\
& \left.\left.+\frac{1}{2} \cdot v_{N_{4}^{+}}\right)+\delta_{A_{r}} \cdot\left(v_{A_{r}}+v_{A_{r}^{+}}+\frac{1}{2} \cdot v_{A_{r_{2}^{+}}}\right)\right]-\left\{\frac{N_{e}}{N_{N_{2}}} \cdot v_{*}\right. \\
& \left.+\left[\frac{1}{\xi_{1}\left(T, N_{N_{2}}\right)}+\left(\frac{N_{A_{r}}}{N_{2}}\right) / \xi_{2}\left(T, N_{A_{r}}\right)\right]\right\} \\
& \left.+\left[\frac{1}{\xi_{1}\left(T, N_{N_{2}}\right)}+\left(\frac{N_{A_{r}}}{N_{2}}\right) / \xi_{2}\left(T, N_{A_{r}}\right)\right]\right\}-\frac{N_{e}}{N_{N_{2}}} \cdot v_{*}^{2} \\
& \wp_{2}=\operatorname{det}(A)=\left\{v_{*}+\left[\delta_{N_{2}} \cdot\left(v_{N_{2}}+v_{N_{2}^{+}}\right)+\delta_{A_{r}} \cdot\left(v_{A_{r}}+v_{A_{r}^{+}}+\frac{1}{2} \cdot v_{A_{r^{+}}}\right)\right]\right\} \cdot\left\{\frac{N_{e}}{N_{N_{2}}} \cdot v_{*}\right. \\
& \wp_{2}=\operatorname{det}(A)=l_{1} \cdot l_{2}-\frac{N_{e}}{N_{N_{2}}} \cdot v_{*}^{2} \\
& +
\end{aligned}
$$

Then

$$
\lambda_{1}=\frac{\wp_{1}+\sqrt{\wp_{1}^{2}-4 \cdot \wp_{2}}}{2} ; \lambda_{1}=\frac{\wp_{1}-\sqrt{\wp_{1}^{2}-4 \cdot \wp_{2}}}{2}
$$

Are the solutions of the quadratic equation (67). The eigenvalues are dependent only on the trace and determinant of the matrix A. The typical case for the eigenvalues is to be distinct $\left(\lambda_{1} \neq \lambda_{2}\right)$. In this case, the theorem states that the corresponding eigenvectors $v_{1}, v_{2}$ are linearly independent, and hence span the entire plane. In particular, any initial condition $x_{0}$ can be written as a linear combination of eigenvectors $x_{0}=\sum_{k=1}^{2} c_{k} \cdot v_{k} \quad$. The general solution for $x(t)$ is $X(t)=\sum_{k=1}^{2} c_{k} \cdot e^{\lambda_{k} \cdot t} \cdot v_{k}$. It is general solution since it is a linear combination of solutions to $\frac{d X(t)}{d t}=A \cdot X(t)$, and is itself a solution. It satisfies the initial condition $X(t=0)=X_{0}$ and the existence and uniqueness theorem give the only solution [7, 8]. The classification of fixed points gives the type and stability of all the different fixed points on a single diagram. The axes are the trace $\wp_{1}$ and the determinant $\wp_{2}$ of the matrix $A$. All of the information in the diagram is implied by the following formulas:

$$
\begin{aligned}
& \lambda_{1,2}=\frac{\wp_{1} \pm \sqrt{\wp_{1}^{2}-4 \cdot \wp_{2}}}{2} ; \wp_{2}=\prod_{k=1}^{2} \lambda_{k} ; \wp_{1}=\sum_{k=1}^{2} \lambda_{k} \\
& \left(\lambda-\lambda_{1}\right) \cdot\left(\lambda-\lambda_{2}\right)=0 \\
& \left(\lambda-\lambda_{1}\right) \cdot\left(\lambda-\lambda_{2}\right)=\lambda^{2}-\lambda \cdot \wp_{1}+\wp_{2}=0
\end{aligned}
$$

If $\wp_{2}<0$, the eigenvalues are real and have opposite sign hence the fixed point is a saddle point. If $\wp_{2}>0$, the eigenvalues are either real with the same sign (nodes), or complex conjugate (spirals and centers). Nodes satisfy $\wp_{1}^{2}-4 \cdot \wp_{2}>0$ and $\quad$ spirals satisfy $\wp_{1}^{2}-4 \cdot \wp_{2}<0$. The parabola $\wp_{1}^{2}-4 \cdot \wp_{2}=0$ is the borderline between nodes and spirals; star nodes and degenerate nodes live on this parabola. The stability of the nodes and spirals is determined by $\wp_{1}$. When $\wp_{1}<0$, both eigenvalues have negative real parts, the fixed point is stable. 
Unstable spirals and nodes have $\wp_{1}>0$. Neutrally stable center lives on the borderline $\wp_{1}=0$, where the eigenvalues are purely imaginary. If $\wp_{2}=0$ then at least one of the eigenvalues is zero. Then the origin is not an isolated fixed point. There is either a whole line of fixed points, or a plane of fixed points, if $A=0$. Saddle points, nodes, and spirals are the major types of fixed points; they occur in large open regions of the $\left(\wp_{2}, \wp_{1}\right)$ plane. Centers, stars, degenerate nodes, and non-isolated fixed points are borderline cases that occur along the curves in the $\left(\wp_{2}, \wp_{1}\right)$ plane $[7,8]$.

\section{Conclusion and results}

In laser system a laser generation in molecular nitrogen in an argon-nitrogen gas mixture is remotely excited at a specific distance above in a femtosecond laser filament. Mid-IR laser pulses enable radical enhancement of fila mentation assisted lasing by $\mathrm{N}_{2}$ molecules. High energies are achieved from laser pulses which generated through the second-positive-band transitions of $\mathrm{N}_{2}$. It is corresponding to a $0.5 \%$ total conversion efficiency from mid infrared laser energy to the energy of UV lasing. Lasing from molecular nitrogen is used in many applications. The discharge-pumped nitrogen laser, operating in a broad range of gas pressures, from several millibars to the atmospheric pressure, and repetition rates from several hertz to several kilohertz. It is robust source of high-power near-UV radiation. Achieving nitrogen via remote excitation would pave the way to many potential applications. A narrow band source of stimulated emission would provide coherent, highly directional radiation for highly selective and sensitive remote spectroscopy of the atmosphere. The possibility for remote ignition of a free localized nitrogen laser is demonstrated previously in a microwave discharge. By using focused laser beams we can excite free-space nitrogen lasing. The process of femtosecond fila mentation in gases is related to remotely excited free-space nitrogen laser. Filamentation of highpower femtosecond laser radiation in gases is connected the formation of a self-guided, highintensity field structure, accompanied by a significant spectral broadening, super continuum generation, and creation of a plasma channel in the wake of the pulse. The femtosecond filaments can be generated at standoff distances of up to tens of kilometers and from ionized channels of up to hundreds of meters. Formation of plasma in the filament, similar to a gas discharge, initiates a chain of plasma chemical reactions in the atmosphere which lead to the appearance of a large variety of neutral and ionic species in rotationally, vibrational, and electronically excited states. We get a situation that favorable conditions for population inversion and lasing between different electronic levels in nitrogen and in oxygen is created. We can use the standoff lasing with a femtosecond filament as an advantage in comparison to scheme due to substantially less scattering and absorption losses.

There is a possibility of stimulated emission from nitrogen in femtosecond filaments. The proof of lasing is based on the exponential fit of the dependence of fluorescence on the filament length. The exponential dependence is valid under the condition of a stationary population inversion only. In filament driven laser the pump is the femtosecond laser pulse and amplified spontaneous emission (ASE) is about nanosecond [1]. The laser generation is built up under the condition of a decaying population inversion which has a decay rate on the same nanoscale time scale. The filament length is calculated from the laser power assuming the direct proportionality between these values. 
Multiple filament formation is expected at high levels of laser power, and the assumption is that the filament length is proportional to the laser power. The generation of micro joule pulses from a cavityfree nitrogen laser which uses the plasma channel traced by a femtosecond laser filament as its active medium. In the lasing process, the electronic transitions of nitrogen are the same as in the conventional discharge-pumped nitrogen laser. In the discharge-driven scheme, the upper lasing triplet $C^{3} \Pi_{u}$ in the three-level nitrogen laser is populated by electron impact excitation from the singlet ground state $X^{1} \Sigma_{g}$. This gives rise to an efficient fluorescence in the near-UV spectral range due to radiative transitions between the vibrational manifolds of $C^{3} \Pi_{u}$ and $B^{3} \Pi_{g}$ states. The control over the density and temperature of electrons is of key importance for obtaining population inversion and lasing between these states. The impulsive RF or capacitor discharge caused to rapid joule heating of electrons due to collisions with neutrals leads to the formation of non-equilibrium energy distribution of electrons. The non-equilibrium energy distribution and the plasma concentration are maintained throughout the discharge, resulting in a sufficient amount of hot electrons - which is needed for efficient pumping. In a laser filament, the electronic energy distribution is formed on the time scale of the femtosecond laser pulse by the optical field ionization process. After the femtosecond pulse, the resultant energy distribution function evolves freely as the plasma can concentration decays. Therefore, the required $C^{3} \Pi_{u}$ excitation by electron impact can be achieved within the average lifetime of the plasma electrons. By using a single femtosecond pulse, we cannot control the electron temperature during the buildup of population inversion, nor to control the duration of the time window within which the nitrogen laser is pumped. The electric discharge pumping, which maintains hot plasma, in the femtosecond filament the optimal electronic energy distribution is governed by optical field ionization and therefore is determined by the intensity and the optical cycle duration. The effect of intensity clamping precluded the possibility of direct scaling of the temperature of electrons in a filament via an increasing of the energy pulse. The plasma density is limited by the self-consistent balance between self-focusing and plasma refraction when focusing conditions are fixed. The coherence length of ASE is determined by the radiative lifetime. We need to inspect and analyze the efficient lasing of molecular nitrogen in a femtosecond filament. In this process the excitation energy of argon atoms transfers to molecular nitrogen. Then, excited argon atoms provide a collisional pump for laser transitions of $\mathrm{N}_{2}$ in a laser-induced filament, as same as hot electrons in a discharge-pumped nitrogen laser [1].

The analytical model is used to analyze the lasing in a laser-induced filament which includes the fila mentation dynamics and of plasma kinetics in the wake of the filament. We get the electron density and the intensity of the laser pulses. The target is to solve the system of plasma kinetics equations jointly with the equations for the electron temperature and the vibrational temperature of ground-state nitrogen molecules. The system is described by the rate equations which are related to electron temperature and the vibrational temperature of ground-state nitrogen molecules. Under some assumptions we get the reduced rate equations. The gas temperature $(T)$ is transform to a new function which is dependent on $T_{\Xi}$ and $\Omega$, $T_{\Xi} \in \mathbb{N} ; \Omega \in \mathbb{R}$, parameters. System fixed points ( 
$\left.T_{v i b r}^{*}, T_{v i b r}^{*}\right)$ are calculated and are dependent on system parameters. The times of vibrational relaxation through $\mathrm{N} 2-\mathrm{N} 2\left(\tau_{V T, N_{2}}\right)$ and $\mathrm{N} 2-\mathrm{A}_{\mathrm{r}}($ $\tau_{V T, A_{r}}$ ) collisions are estimated using the LandauTeller approximation. System reduced rate equations linearization is done and calculated characteristic equation.

System stability and stability switching is inspected under parameters variation, and we implied the stability behavior by eigenvalues expression (trace and determinant elements). By inspecting the trace and determinant elements we can detect the stability state of our system. We categorized system fixed points by eigenvalues to saddle point, nodes, spiral, and centers (oscillatory system), star nodes and degenerate nodes. We additionally detect a whole line of fixed points, or a plane of fixed points.

The system critical parameters are the key element which establish the trace $\left(\wp_{1}\right)$ and determinant ( $\wp_{2}$ ) value and then the stability state of our system $[7,8]$. Upon changing the gas temperature $(T)$ values some interesting behavior is inspected in our nitrogen gas laser filament plasma kinetic system.

\section{REFERENCES}

1. Aluf O. Advance Elements of Laser Circuits and Systems: Non-linearity Application in Engineering, Springer (2021).

2. Daniel K, Skimantas A, et al. Free-space nitrogen gas laser driven by a femtosecond filament. Phys. Rev. A 86 (2012): 033831.

3. Shneider MN, Baltuska A, Zheltikov AM. Population inversion of molecular nitrogen in an $\mathrm{Ar}: \mathrm{N}_{2}$ mixture by selective resonance enhanced multiphoton ionization, Journal of Applied Physics 110 (2011): 083112.

4. Beretta E, Kuang Y. Geometric stability switch criteria in delay differential systems with delay dependent parameters, SIAM J. Math. Anal 33 (2002): 11441165 .

5. Hale JK. Dynamics and Bifurcations. Texts in Applied Mathematics 3 (1996).

6. Betta E, Kuang Y. Geometric stability switch criteria in delay differential systems with delay dependent parameters, SIAM J. Math. Anal 33 (2002): 11441165 .

7. Steven H. Strogatz, Nonlinear Dynamics and Chaos: With Application to Physics, Biology, Chemistry, and Engineering (Studies in Nonlinearity), CRC Press; $1^{\text {st }}$ edition (2000).

8. Guckenheimer J, Holmes P. Nonlinear Oscillations, Dynamical Systems, and Bifurcation of Vector Fields, SpringerVerlag (1983): 453.

9. Fan Rongwei, Zhu Hai, Li Xiaohui, et al. Characterization of Laser Induced Plasma in Nitrogen, Journal of Russian Laser Research 34 (2013).

10. Avizonis PV, Dean DR, R Grotbeck. Determination of vibrational and translational temperatures in gas-dynamic las-ers, Appl. Phys. Lett 23 (1973).

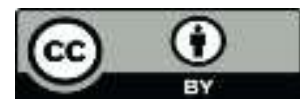

This article is an open access article distributed under the terms and conditions of the Creative Commons Attribution (CC-BY) license 4.0 